A ten-year-old boy was referred to the Clinic for further treatment after frequent bronchopneumonia in the last 4 years. Each time on the X-ray, basal right, the shadow on the lungs was verified. After antibiotic therapy, the control X-ray always showed incomplete regression of the shadow. The boy was born with esophageal atresia with tracheoesophageal fistula and was successfully operated on the eighth day of life.

Post-operative dilatation of esophagus has been successfully performed twice. The boy was in a good general health condition, except he was often tired after the activity. With the auscultation of the lungs, basal right, crepitation could be heard. CT of the chest, in the area of the posterobasal segment of the right lower lung lobe, showed a cluster of cystic air forms with condensed pulmonary parenchyma. The described change corresponded to developmental lung anomaly - cystic adenomatoid lung malformation. Right thoracotomy and lobectomy of the right lower lung lobe were performed. By surgery, the boy had no recurrent bronchopneumonia. Approximately one-third of CPAMs are diagnosed after the neonatal period. These lesions typically are CPAM types 1, 2 or 4 , and tend to be smaller than CPAMs that present with respiratory symptoms at birth. A common presentation in older children is recurrent pneumonia. Other presenting complaints include cough, dyspnea and/or cyanosis. Findings on physical examination include decreased breath sounds over the lesion, hyperresonance and chest wall asymmetry with a bulge on the affected side.

\section{SUCCESSFUL TREATMENT OF PRIMARY SPONTANEOUS PNEUMOTHORAX WITH VIDEO-ASSISTED THORACOSCOPIC PLEURECTOMY}

Marko Bašković* ${ }^{*}$ Josip Pejić, Ljudevit Sović, Dora Škrljak Šoša, Mirko Žganjer, Ante Čizmić. Children's Hospital Zagreb

\subsection{6/archdischild-2021-europaediatrics.419}

Pneumothorax is defined as a collection of air that is located between the visceral and parietal pleura. Spontaneous pneumothorax occurs in the absence of any identified trauma. It is subdivided into primary and secondary types. The annual incidence of primary spontaneous pneumothorax (PSP) in the general population is estimated to be 5 to 10 per 100000 . The peak incidence occurs between 16 and 24 years of age. We present the case of a sixteen-year-old boy who manifested on several occasions with right-sided spontaneous pneumothorax. The boy was actively involved in sports. Until spontaneous pneumothorax occurred, he had never been ill. He did not take medication. He had no allergies. A total of three times the right chest was drained. At each arrival, pneumothorax manifested with abrupt pain. The X-ray confirmed the diagnosis each time. After a third spontaneous pneumothorax, a CT of the chest was done. CT showed small apical blebs. The trachea was neatly positioned, neatly branching. The right main bronchus seemed wider than the left. The vascular structures were of a neat appearance. The heart was neatly positioned, with a morphologically neat appearance. Surgical treatment was initiated. After collapse of the right lung, an incision was made in the third intercostal space to the right in the anterior axillary line. An angle camera was installed. Upon placement of the camera, the trocar is placed in the seventh intercostal space in the median axillary line. After identifying the apical blebs changes with a linear endostapler, a resection is performed. A partial parietal pleurectomy is then performed. Negative pressure drainage is set. In prophylaxis, the boy received cefazolin. A one-year follow-up did not result in relapse.

Surgery for pneumothorax consists of stapling ruptured blebs and resection of abnormal lung tissue. The approaches used include VATS (video-assisted thoracoscopic surgery), mini-thoracotomy and conventional thoracotomy. We usually use VATS, which provides adequate exposure for resection or stapling and an opportunity for pleurectomy, abrasion or chemical pleurodesis. The morbidity of VATS is less than with conventional or mini-thoracotomy, and recurrence rates are approximately 5\%, although open thoracotomy and pleurectomy have the lowest recurrence rate. Given that in our previous cases, after repeated tube drainage, we did not have recurrences, we did not opt for VATS immediately after the first recurrence. Reading the literature, in the future we will be guided by the algorithm to initiate VATS after the first recurrence of pneumothorax.

\section{INTERSTITIAL LUNG DISEASE DUE TO SFTPC MUTATION - A RARE CASE REPORT}

Anupama Mallappa*, Catriona Mary Middleton. Royal Aberdeen Children's Hospital

10.1136/archdischild-2021-europaediatrics.420

Interstitial Lung Disease due to SFTPC mutation -a rare case report Mutations of the surfactant protein (SP)-C gene SFTPC have been associated with neonatal respiratory distress syndrome and interstitial lung disease.

If accurate diagnosis and proper management are delayed, irreversible respiratory failure may occur. A girl born following dizygotic twin pregnancy born at $37+3$ weeks, born in good condition, no active resuscitation. However noted to have a fast-respiratory rate. Subsequently has had several admissions to hospital with faltering growth, persistent tachypnoea and oxygen requirement. High resolution CT angiogram showed atelectatic changes in the dependant portions, genetics work up identified a variation in a gene called SFTPC.

Introduction Pulmonary surfactant is a complex mixture of phospholipids and proteins that reduces surface tension of the alveolus by forming a surface-active film at the air-liquid interface. Of the four surfactant associated proteins the hydrophobic SP-B and ASP-C are especially essential in the dynamics of phospholipids by promoting the transition between the storage form and the functional surface film.

SP-C is synthesized from a 197 -amino acid precursor (proSP-C )which is encoded by SFTPC on human chromosome $8 \mathrm{p} 21.3$. In this report we present a case of a child who has childhood interstitial lung disease due to SFTPC mutation (c.289G > T).

Child Details A 5-week-old initially presented with possible aspiration; she was noted to be persistently tachypneic (up to 90 breaths/min) needing minimal oxygen to maintain saturations. She was reviewed weekly, where she remained tachypneic associated with feeding issues and faltering growth, was extensively investigated (GI/cardiac/metabolic). Had a prolonged stay at hospital and was subsequently discharged home on $0.5 \mathrm{~L} \mathrm{O} 2$, high calorie $\mathrm{NJ}$ feeds and anti-reflux medications (post barium study).

Over the course she has had several admissions to the hospital, she has been treated with methylprednisolone 6 doses, 